\title{
First Results of Cavitation Erosion Behavior of Plasma Nitrided Niobium: Surface Modification
}

\author{
Ricardo Kertscher ${ }^{a}$, Juliana Martins de Moraes ${ }^{a}$, Sérgio Henke ${ }^{a}$, Angela Nardelli Allenstein ${ }^{b}$, \\ Regis Henrique Gonçalves e Silvac, Jair Carlos Dutrac, Silvio Francisco Brunatto ${ }^{a *}$ \\ ${ }^{a}$ Laboratório de Tecnologia de Pós e Plasma - LT2P, Departamento de Engenharia Mecânica, \\ Universidade Federal do Paraná - UFPR, Av. Cel. Francisco H. dos Santos, 210, \\ Jardim das Américas, CEP 81531-980, Curitiba, PR, Brasil \\ ${ }^{b}$ SpectroScan Tecnologia de Materiais Ltda., R. Wanda dos Santos Mullmann, 1233, \\ CEP 83323-400, Pinhais, PR, Brasil \\ ${ }^{c}$ Laboratório de Soldagem - LABSOLDA, Departamento de Engenharia Mecânica, \\ Universidade Federal de Santa Catarina-UFSC, Bloco B, Campus Universitário, \\ $s / n$, Trindade, CEP 88040-900, Florianópolis, SC, Brasil
}

Received: July 28, 2015; Revised: August 24, 2015

\begin{abstract}
This work presents the first results of the plasma nitriding study performed in pure niobium in order to increase its cavitation erosion resistance. Samples were prepared from $98.9 \%$ purity and $90 \%$ reduction cold-rolled niobium bars. Annealing treatment of the cold-worked niobium samples was carried out in vacuum furnace at $1.33 \mathrm{~Pa}$ pressure, in the temperature of $1000^{\circ} \mathrm{C}$, for a time of $60 \mathrm{~min}$. Annealed samples showing hardness of $80 \mathrm{HV}$ were cut to dimensions of $20 \times 30 \times 4 \mathrm{~mm}^{3}$. Nitriding treatment was conducted at $1080{ }^{\circ} \mathrm{C}$, gas mixture of $90 \% \mathrm{~N}_{2}+10 \% \mathrm{H}_{2}$, flow rate of $5 \times 10^{-6} \mathrm{Nm}^{3} \mathrm{~s}^{-1}$, and pressure of $1200 \mathrm{~Pa}$ (9 Torr), for a total time of $4 \mathrm{~h}$ comprised by two treatment steps of $2 \mathrm{~h}$ each. For comparison purpose, results for nitrided and non-nitrided niobium are confronted. Samples were characterized by XRD, nanoindentation, microhardness, SEM, and 2D surface topography and 3D interferometry profile analysis techniques. Cavitation testing was conducted according to ASTM G32-09. Comparatively, promising results based on the formation of niobium nitride phases in treated surfaces are presented and discussed in the present work.
\end{abstract}

Keywords: cavitation-erosion, niobium nitride, plasma nitriding, niobium

\section{Introduction}

Cavitation erosion of metals is characterized by local impacts of fluid against the metallic surface due to collapse of bubbles, resulting in progressive loss of original material from the solid surface, in the vicinity of the active cloud within a liquid. Some problems related to cavitation are vibration in hydraulic machines, increase in hydrodynamic drag and change in hydrodynamic flow, noise, thermal and light effects like luminescence, besides erosion wear ${ }^{1}$. Erosion wear due to cavitation phenomenon has demanded special attention of researchers and engineers mainly in power generation plants. This is due to the high costs associated to preventive and corrective maintenance of hydraulic machine components, which are continually exposed to cavitation. The relative resistance of materials to cavitation erosion is commonly determined by mass loss measurement in laboratory, and it has been reported in terms of two quantities, the nominal incubation period (IP) and the maximum erosion rate (MER) ${ }^{2}$.

In previous work ${ }^{2}$, cavitation erosion behavior of pure niobium was investigated by means of a $20 \mathrm{kHz}$ ultrasonic vibrator at peak-to-peak amplitude of $50 \mu \mathrm{m}$, aiming to determine the niobium potential as a material for the

*e-mail: brunatto@ufpr.br manufacturing of hydraulic machine components. The study was emphasized for the three first cavitation stages of the cumulative erosion-time curve. The modification of the niobium surface morphology as a function of the cavitation testing time in the incubation, acceleration, and maximum erosion rate stages was verified for niobium samples in cold-worked and annealed conditions. Results indicated that the annealing treatment leads the niobium hardness to be decreased and its ductility to be increased, resulting to an increase of the incubation period when compared with the cold-worked niobium. Finally, it was concluded that the cavitation erosion failure mechanism in annealed niobium occurs in a sequence of events comprising the work-hardening effect and the fracture of debris allied to the effect of fatigue and microcracks formation.

On the other hand, plasma nitriding has been widely used in many industrial applications for different materials, aiming to improve specific properties of treated surfaces, such as wear and fatigue resistance, surface hardness, corrosion resistance, and eventually to obtain reduced static friction coefficient between two solid surfaces. In recent work ${ }^{3}$, plasma nitriding of pure niobium was investigated using a DC square waveform pulsed power supply of $5.0 \mathrm{~kW}$ for 
the temperature range of $500-1080{ }^{\circ} \mathrm{C}$, aiming to change the material surface characteristics due to heating and physical-chemical reactions caused by the ions and fast neutral bombardment during the treatment. Strong increase of the surface hardness varying on the order of $2.5 \mathrm{GPa}$ for pure niobium (as non-nitrided) to $21 \mathrm{GPa}$ in the as-nitrided condition was verified, being this result attributed to the formation of $\varepsilon-\mathrm{NbN}$ and $\beta-\mathrm{Nb}_{2} \mathrm{~N}$ hexagonal phases in the nitrided layer. In addition, significant changes on surface morphology and roughness of the treated samples were observed for the studied conditions and such results were discussed considering the sputtering and plasma species bombardment effect.

It is worth to mention that nitriding techniques have been successfully applied, mainly for different stainless steels, aiming to increase their cavitation erosion resistance. In prior work ${ }^{4}$, improvement of the cavitation erosion resistance was verified by applying the high temperature gas nitriding technique for an AISI 304L austenitic stainless steel. In more recent work ${ }^{5}$, similar result was verified, but in this case, by using the low-temperature plasma nitriding technique in a CA-6NM martensitic stainless steel. In both the cases $^{4,5}$, the gain on the cavitation erosion resistance was attributed to nitrogen-expanded austenite formation in the nitrided layers. But, in the specialized literature, cavitation erosion behavior of plasma nitrided niobium has not been studied. Despite that significant research works have been done on the cavitation behavior of pure metals and metallic alloys, a much lesser degree of published works has studied the behavior of engineering ceramics ${ }^{6-13}$. Plasma nitriding technique was used here in order to obtain niobium nitride (ceramic-like material) surfaces formed in pure niobium substrates. Thus, the cavitation erosion behavior of the obtained system 'ceramic layer surface + metallic bulk' is the main subject of the present work. Potential applications comprise the cavitation erosion problem in high temperature materials, like those used in gas turbines. In this entry, the reader is then firstly directed to aspects related to cavitation erosion behavior of structural ceramics, since the cavitation erosion behavior of ceramic materials are quite different from that of metals. The morphology and roughness changes of the nitrided surface, due to ions and fast neutrals bombardment is carefully characterized, considering that surface properties are determinant in cavitation erosion behavior of different substrates. Then, the new material formed at the plasma nitrided surface, typically a ceramic, is characterized in terms of its structure and mechanical properties, before being subjected to the cavitation erosion testing study.

\section{Cavitation Erosion Behavior of Structural Ceramics}

Some important aspects of the cavitation erosion behavior of structural ceramics are presented in this section, aiming to base the main events that can be expected to occur when plasma nitrided niobium samples are subjected to cavitation erosion testing.

In a previous work ${ }^{6}$, aspects which can lead structural ceramics to present high cavitation erosion resistance are discussed. Results clearly show that surface finishing critically affects the incubation period, being that the selection of the correct material with a suitable surface preparation can increase the incubation period by over $1000 \%{ }^{6}$. Ceramic with high resistance to cavitation erosion in terms of both the incubation period and erosion rate would have ultrafine microstructure, no porosity, high fracture toughness and a smooth surface finish, as a whole ${ }^{6}$. It was also observed by studying different ceramic materials that the possibility of exploring compressive stresses in surface layers would be an interesting prospect to improve the incubation period ${ }^{6}$.

As presented $i^{8}$, silicon nitride and zirconia has been reported as the technical ceramics with the best cavitation erosion resistance in the literature ${ }^{6-8}$, comprising the main subject for studies aiming to improve the performance of bearings, injectors or valves submitted to cavitation problem in real and different operating conditions, such as those verified in rocket engines ${ }^{8,10,11}$. Karunamurthy et al. have also shown that cracking in cavitation eroded silicon nitride is preferentially intergranular, leading erosion pits to be produced. The presence of microcracks at the pits boundaries would result in a coalescence mechanism or pits bridging formation, leading more pits to be successively removed as the surface exposition to cavitation erosion goes on ${ }^{10}$.

Finally, possible deformation and cracking mechanisms in the pit formation are presented in a study of cavitation exposure carried out in silicon nitride and zirconia $^{8}$. In general terms, according to the authors ${ }^{8}$, it has been established that pseudoplastic deformation pits are formed when tensile stress induced by the impact of the collapsing bubble, leading the surface to be elastically deformed, is high enough to create cracks in intergranular phase next to the surface. These cracks release the slip systems in the crystals, making possible the occurrence of a small plastic deformation, as a result of the water pressure action, within the remaining grains after the impact ${ }^{8}$. Local plastic deformation tends to occur at the same time as the surface is still highly elastically deformed, being that the plastically deformed surface fits the new geometry of the elastically deformed bulk material ${ }^{8}$. After the pressure action from the collapsing bubbles ends, the bulk material tends to return to its original geometry, but since the new surface does not match that geometry, a residual stress state is created, leading the pit to be formed ${ }^{8}$. For the case that cracks are not created, the surface simply would return elastically to its original geometry ${ }^{8}$.

The different aspects evidenced in structural ceramic surfaces subjected to cavitation erosion testing, as above presented, comprise a list of possible events that could be also observed in nitrided surfaces exposed to cavitation testing, in the present work, considering the formation of niobium nitride phases at the niobium substrates. But, as it will be discussed hereafter, the main difference in this case is that the ceramic phase, supposedly formed at the substrate surface, comprises a nitrided layer obtained by nitrogen diffusion into a metallic bulk of the niobium substrate. So, the system 'surface + bulk' to be considered in this work would be formed by 'ceramic layer + metallic bulk', thus differing from those 'ceramic surface + ceramic bulk' as previously shown in references ${ }^{6-11}$.

To finish, it is worth to be noted that the system to be studied here also differs substantially from those in ${ }^{12-13}$, as 
for example, for thin films deposited on substrate surfaces, for which brittle mode fracture and film delamination would be present $t^{13}$. As expected for that case of thin film coatings, independently of the substrate nature, if ceramic or metallic, adhesion should be a decisive property to be considered, which concludes the main aspects related to the present subject.

\section{Experimental Procedure}

Niobium samples were cut by wire electrical discharge machining (WEDM) in dimensions of $30 \times 20 \times 4 \mathrm{~mm}^{3}$ from $98.9 \%$ purity and $90 \%$ reduction cold-rolled niobium bar. Annealing treatment of the cold-worked niobium samples was carried out in vacuum furnace at $1.33 \mathrm{~Pa}\left(10^{-2}\right.$ Torr $)$ pressure, in the temperature of $1000^{\circ} \mathrm{C}$, for a time of $60 \mathrm{~min}$. The samples were ground using 220, 320, 400, 600 and $1200 \mathrm{SiC}$ sand-paper. Aiming to obtain the finishing of the testing surfaces, samples were initially polished using $1.0 \mu \mathrm{m}$ diamond paste, and finally polished with $0.05 \mu \mathrm{m} \mathrm{Al}_{2} \mathrm{O}_{3}$ solution. The etchant ASTM 160(a), composed of $20 \mathrm{ml} \mathrm{HF}$, $15 \mathrm{ml} \mathrm{H}_{2} \mathrm{SO}_{4}, 5 \mathrm{ml} \mathrm{HNO}, 50 \mathrm{ml} \mathrm{H}_{2} \mathrm{O}$, applied during $5 \mathrm{~min}$ by sample immersion, was used to reveal the nitrided layer and its thickness. The annealed niobium structure (the grain boundary) was revealed using the etchant ASTM 160 SWAB $15 \mathrm{Ss}$, composed of $40 \mathrm{ml} \mathrm{HF}, 30 \mathrm{ml} \mathrm{H}_{2} \mathrm{SO}_{4}, 10 \mathrm{ml} \mathrm{HNO}_{3}$, $100 \mathrm{ml}$ de $\mathrm{H}_{2} \mathrm{O}$, during $15 \mathrm{~s}$ application at the polished surface.

Plasma nitriding was conducted at $1080{ }^{\circ} \mathrm{C}$, gas mixture of $90 \% \mathrm{~N}_{2}+10 \% \mathrm{H}_{2}$, flow rate of $5 \times 10^{-6} \mathrm{Nm}^{3} \mathrm{~s}^{-1}(300 \mathrm{sccm})$, and pressure of $1200 \mathrm{~Pa}$ (9 Torr). The total nitriding time of $14.4 \mathrm{ks}(4 \mathrm{~h})$ was achieved by two treatment steps of $7.2 \mathrm{ks}$ ( $2 \mathrm{~h}$ ) each, intercalated by the vacuum chamber cooling up to room temperature. Treatments were carried out using $4.16 \mathrm{kHz}$ square-wave pulsed DC power supply, at a peak voltage of $670 \pm 15 \mathrm{~V}$, and pulse period of $240 \mu \mathrm{s}$. The sample temperature was controlled by varying the duty cycle, and it was measured by means of a chromel-alumel thermocouple (type $\mathrm{K}$, and $1.5 \mathrm{~mm}$ diameter), which was inserted to a depth of $15 \mathrm{~mm}$ inside the sample. Before nitriding step, sample was cleaned using $\mathrm{H}_{2}$ glow discharge at $300{ }^{\circ} \mathrm{C}$, for $0.6 \mathrm{ks}$ (10 $\mathrm{min})$, and pressure of $400 \mathrm{~Pa}$ (3 Torr). After this step, the gas pressure was adjusted to the specified value of $1200 \mathrm{~Pa}$ (9 Torr), and the sample was heated up to the nitriding temperature by plasma species bombardment at a heating rate on the order of $0.32{ }^{\circ} \mathrm{Cs}^{-1}$.

The $30 \times 20 \mathrm{~mm}^{2}$ sample surfaces were characterized by SEM, XRD, nanohardness, microhardness, and high precision $3 \mathrm{D}$ interferometry profile analysis techniques. Measurements of surface finishing were performed in accordance with ISO 25178, using a Talysurf CCI - Lite Non-contact 3D Profiler, from Taylor Hobson. In this case, measurements were performed before and after plasma nitriding treatment, using a area of $1.5 \times 1.5 \mathrm{~mm}^{2}$, with a resolution of $1632 \mu \mathrm{m}$, $1633 \mu \mathrm{m}$, and $0.01 \mathrm{~nm}$, for $\mathrm{X}, \mathrm{Y}$, and $\mathrm{Z}$ axis, respectively. The average and standard deviation values of the surface roughness parameters $S a, S z, S p, S v, S d s, S s k$, and $S k u$, here presented, were obtained by analyzing six measure areas per sample, being realized three characterizations on each sample side. It is to be noted that $3 \mathrm{D}$ roughness parameters give more significant values than the $2 \mathrm{D}$ ones, which are achieved by analyzing the roughness profile on a rope.
According to standard ISO 25178, 3D parameters $S a, S z$, $S p, S v, S d s, S s k$, and $S k u$, can be understood as follows:

i) $\quad \mathrm{Sa}=$ the average (arithmetic mean) or the absolute values for the peaks height and valleys depth in the measured area;

ii) $S z=$ the mean of the maximum height of the surface, or the height between the highest peak and the deepest valley;

iii) $S p=$ the maximum peak height, or the height between the highest peak and the medium plane;

iv) $S v=$ the maximum valley depth, or the deep between the deepest valley and the medium plane;

v) $S d s=$ the density of summits. This parameter is expressed in peaks $/ \mathrm{mm}^{2}$. It takes into account that a determined point is considered a peak if it is higher than its 8 neighbors;

vi) $S s k=$ the skewness. Skewness equal to zero means a symmetrical height distribution in relation to the average line, for eventual occurrence of deep valleys or high peaks; skewness positive ( $S s k>0$, or skewed downward in relation to the average line), is typical for surfaces with high peaks and/or filled valleys; and skewness negative ( $S s k<0$, or skewed upward in relation to the average line) tends to represent surfaces with deep scratches and/or removed peaks; and, finally,

vii) $S k u=$ the kurtosis. Kurtosis equal to 3 reflects in a normal probability density curve in terms of the surface roughness height distribution; for $\mathrm{Sku}>3$, the curve height distribution in relation to the average line is spiked, that means the occurrence of many high peaks and many deep valleys; in the other hand, for $\mathrm{Sku}<3$, the form of the surface roughness height distribution is squashed, that means the occurrence of few high peaks and few deep valleys.

Vickers microhardness characterization of the studied surfaces was performed using a Shimadzu Micro Hardness Tester HMV2T, for applied loads of 0.5 (50 gf), 1 (100 gf), and $2(200 \mathrm{gf}) \mathrm{N}$, and peak-load contact of $15 \mathrm{~s}$. The presented hardness values correspond to a mean of four measurements, in each case. Nanoindenter XP-MTS System was also used to determine the hardness and to perform scratch testing and roughness analysis of the studied surfaces. The hardness versus penetration depth curves were obtained on the average of 25 (5 $\times 5$ matrix) indentations with $100 \mu \mathrm{m}$ spacing, using a Berkovich type indenter, and 12 charge-discharge cycles, for loads up to $400 \mathrm{mN}$ (40 gf) and $10 \mathrm{~s}$ loading time. The scratch tests were performed using loadings from 0 to $400 \mathrm{mN}$ ( 0 to $40 \mathrm{gf}$ ), scratch length of $600 \mu \mathrm{m}$ and indenter translation velocity of $10 \mu \mathrm{ms}^{-1}$. Topography profiles at the studied surfaces were also obtained, by making the indenter tip to run along $0.5 \mu \mathrm{m}$ length.

The identification of the phases present in the studied surfaces was carried out by the X-Ray Diffraction (XRD) technique, using a Shimadzu XRD 7000 X-ray diffractometer, 
with $\mathrm{CuK}_{\alpha}$ radiation, at Bragg-Brentano configuration, for $2 \theta$ angles ranging from 30 to $120^{\circ}$, and scanning speed of $0.01^{\circ} \mathrm{s}^{-1}$. On the other hand, Scanning Electron Microscopy (SEM) analysis was performed aiming to determine the surface morphology of the studied samples and the characteristics of the cavitation eroded surfaces, using two distinct equipments, in the case one from JEOL and other from Zeiss.

The cavitation experiment as a function of testing time was conducted on nitrided and non-nitrided niobium surfaces, using a standard cavitation erosion tester, according to ASTM G 32-09. Samples were tested via indirect method, using an ultrasonic generator KLN type 500. The vibratory frequency and the peak-to-peak amplitude were $20 \pm 2 \mathrm{kHz}$ and $50 \pm 1.0 \mu \mathrm{m}$, respectively. The tip of the probe was machined from AISI 304 stainless steel and polished for $1.0 \mu \mathrm{m}$ surface roughness. The temperature of the distilled water bath was maintained at $25 \pm 2{ }^{\circ} \mathrm{C}$. The surface of the test specimen was immersed $12 \mathrm{~mm}$ in distilled water, distant $0.5 \pm 0.005 \mathrm{~mm}$ from the probe tip. The cumulative mass loss of the test specimens was determined by means of $0.1 \mathrm{mg}$ precision balance, for testing times up to $960 \mathrm{~min}$ (16 h). The cavitation erosion behavior results are discussed in terms of the three first cavitation stages of the cumulative erosion-time curves. Experiments were repeated to confirm the reproducibility and to determine the dispersion range of the obtained results.

\section{Results and Discussion}

\subsection{Characterization of plasma nitrided niobium surface}

SEM micrograph of the surface aspect of non-nitrided and nitrided niobium is presented in Figure 1a, b, respectively. Figure la shows the initial condition of the surface obtained by mechanical polishing before being subjected to plasma nitriding treatment. Significant change of the polished surface morphology (Figure 1a) is observed by comparing with that of the plasma nitrided sample (Figure 1b). The changes in the surface morphology are due to the different phenomena of the plasma-surface interaction occurring in plasma nitriding, leading the nitrided layer to be formed at the treated surface, in the case, sputtering, scattering and backdiffusion in gas phase of sputtered metal atoms, with eventual subsequent condensation at the original surface. Formation of clusters of condensed metal atoms and nitrogen at the surface tends to result in island-like products nucleated at the surface, at the same time in which the nitrided layer is formed by diffusion of nitrogen into the substrate bulk. Such island-like products nucleated at the surface can be easily seen in Figure 1b, and they are responsible by the morphology modification observed on the plasma nitrided surface. The surface morphology noted for the nitrided sample (Figure 1b) also remembers the presence of atom islands growing at the surface, as presented $\mathrm{in}^{3}$. In that case $\mathrm{e}^{3}$, the mechanism of surface modification was supposedly due to sputtering and re-condensation of atoms on the surface, resulting in the formation of atom islands and/or clusters at the surface, in similar way to the verified in thin film formation, according to Chapman ${ }^{14}$. The increment of the surface finishing parameters observed for the studied

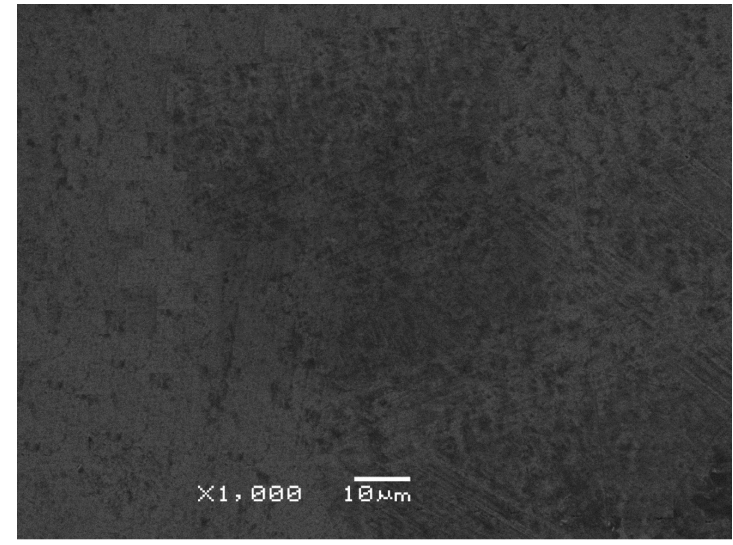

(a)

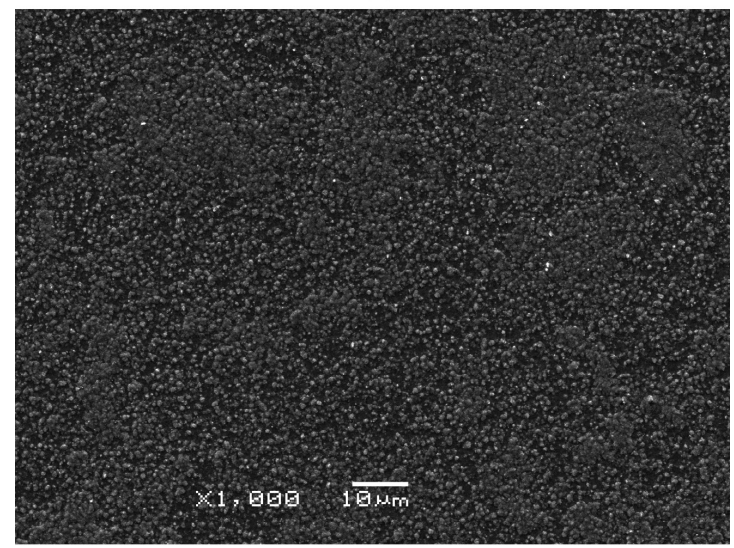

(b)

Figure 1. SEM micrograph of the surface aspect of: (a) non-nitrided; and (b) nitrided niobium.

nitrided samples agrees very well with the formation of sub-micrometric atom islands at the treated surface.

The assertion with regard to islands-like products nucleated at the nitrided surface is based on the 2D topography profile results presented in Figure 2. It is shown in Figure 2 that the number of peaks with amplitude between 0 and $+600 \mathrm{~nm}$ at the nitrided surface was strongly increased in relation to the valleys (which ranged between 0 and $-400 \mathrm{~nm}$ ), comparatively to the non-nitrided surface. This is also confirmed by Table 1 results, which shows the average and standard deviation values of the 3D surface roughness parameters $S a, S z, S p, S v, S d s$, $S s k$, and $S k u$. According to Table 1, $S a, S z, S p, S v$, and $S d s$ values of the nitrided surface were increased of 5.2, 4.0, 6.5, 2.7 , and 1.8 times in relation to non-nitrided surface, all these roughness parameters confirming the assertion regarded to the islands-like products nucleated at the nitrided surface, which make the surface roughness to be grown. Despite that the verified roughness increment in the nitrided surface could be detrimental for cavitation erosion purposes, since it is very well established that roughness surface and cavitation resistance tend to be inversely correlated greatness, it is to be considered that the referred roughness increment is a self-result of the plasma nitriding process, and additional efforts to be conducted are necessary aiming to overcome this supposed problem. 
In addition, considering the $S s k$ results, the negative value $(-0.85)$ verified at the non-nitrided surface tends to confirm the occurrence of a surface presenting deep scratches, and/or removed peaks, agreeing well with the initial condition of the studied surface which was achieved by polishing. On the other hand, the skewness at $4 \mathrm{~h}$ plasma nitrided surface was changed to a positive value $(+0.02)$, clearly indicating a surface presenting high peaks, and/or filled valleys. It is to be noted that the $S s k$, and $S p$ results of the nitrided surface also agree with the islands-like morphology shown in Figure 1b, by comparing with the results of the non-nitrided surface. To finish, the $S k u$ values verified for both the non-nitrided and nitrided surfaces were higher than 3. For $S k u>3$, the curve height distribution in relation to the average line is spiked, that means the occurrence of many high peaks and many deep valleys. It is also presented in Table 1, 3D surface roughness parameters results of repolished-after-nitrided

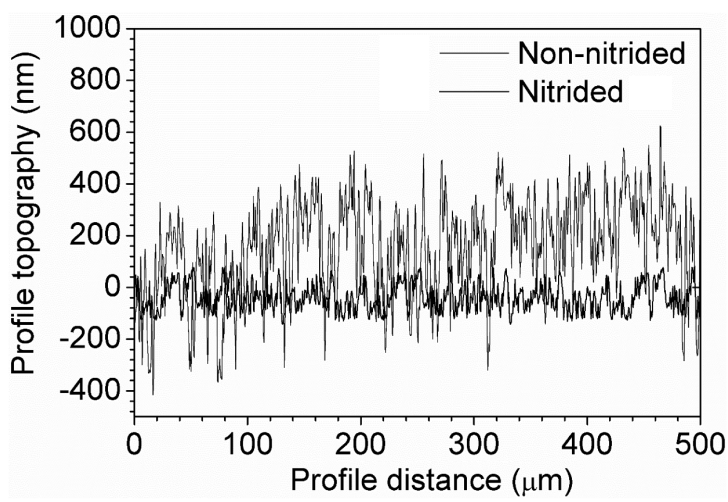

Figure 2. 2D topography profile results of the non-nitrided and nitrided niobium surface.

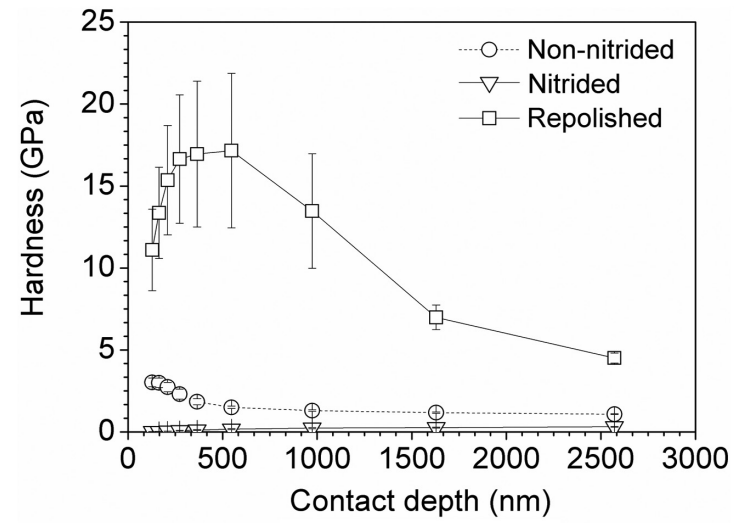

(a) surface. Repolishing procedure of the nitrided surface was necessary since the high roughness verified for the nitrided surface makes impossible the hardness characterization by nanoindentation technique, as verified ahead (see Figure 3 results).

In addition, it is shown in Figure $4 \mathrm{a}, \mathrm{b}$, cross-section SEM micrographs of the niobium before nitriding, and the aspect of the nitrided layer obtained after $4 \mathrm{~h}(2+2 \mathrm{~h})$ nitriding treatment, respectively. A typical full annealed niobium microstructure can be observed in Figure 4a, which is characterized by equiaxed grains present all over the niobium substrate. In Figure 4b, nitrided layer thickness measured values attained by SEM observation are shown, confirming that the nitriding procedure used in present work has led to the obtainment of nitrided layers on the order of $3.3 \mu \mathrm{m}$ in depth. It is to be noted that both the Figures $1 \mathrm{~b}$ and $4 \mathrm{~b}$ correspond to the same specimen, and the evidence of islands formed on the surface as well as the eventual presence of craters between the coalesced islands, in Figure $4 \mathrm{~b}$, depends exclusively on the position of the cut at the cross-section, during the sample preparation stage. Finally, the XRD patterns of the nitrided and non-nitrided surface studied in present work are shown in Figure 5. Results indicating the presence of $\mathrm{NbN}-\varepsilon$ and $\mathrm{Nb}_{2} \mathrm{~N}-\beta$ phase layer confirm the formation of typical ceramic material in the nitrided surface, being in agreement with earlier results presented in $^{3}$.

\subsection{Mechanical characterization of the nitrided layer}

Figure 6 shows hardness values of the studied surfaces as a function of the load obtained by using the Vickers indentation technique. The relatively constant measured values, on the order of $85 \mathrm{HV}$ for non-nitrided surfaces are in agreement with the expected for the annealed pure

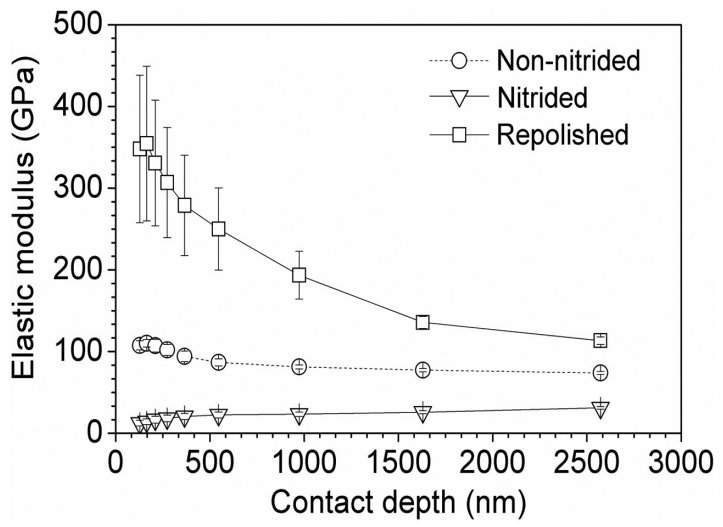

(b)

Figure 3. (a) Nanohardness; and (b) Elastic modulus results as a function of the indentation (penetration) depth, for the studied surfaces.

Table 1. Average (+ standard deviation) values for the surface roughness parameters $S a, S z, S p, S v$, $S d s$, and $S s k$, obtained at non-nitrided, nitrided, and repolished-after-nitrided surfaces.

\begin{tabular}{lccccccc}
\hline \multicolumn{1}{c}{ Condition } & $\mathbf{S a}(\boldsymbol{\mu m})$ & $\mathbf{S z}(\boldsymbol{\mu m})$ & $\mathbf{S p}(\boldsymbol{\mu m})$ & $\mathbf{S v}(\boldsymbol{\mu m})$ & Sds $\left(\mathbf{1} / \mathbf{m m}^{\mathbf{2}}\right)$ & \multicolumn{1}{c}{ Ssk } & Sku \\
\hline Non-nitrided & $0.05(0.06)$ & $0.60(0.07)$ & $0.20(0.01)$ & $0.41(0.06)$ & $10900(445)$ & $-0.85(0.17)$ & $5.45(1.72)$ \\
Nitrided & $0.26(0.02)$ & $2.42(0.22)$ & $1.31(0.25)$ & $1.10(0.05)$ & $19700(750)$ & $0.02(0.15)$ & $3.45(0.41)$ \\
Repolished & $0.11(0.01)$ & $1.08(0.07)$ & $0.51(0.08)$ & $0.61(0.06)$ & $25117(1111)$ & $-0.27(0.06)$ & $3.81(0.29)$ \\
\hline
\end{tabular}




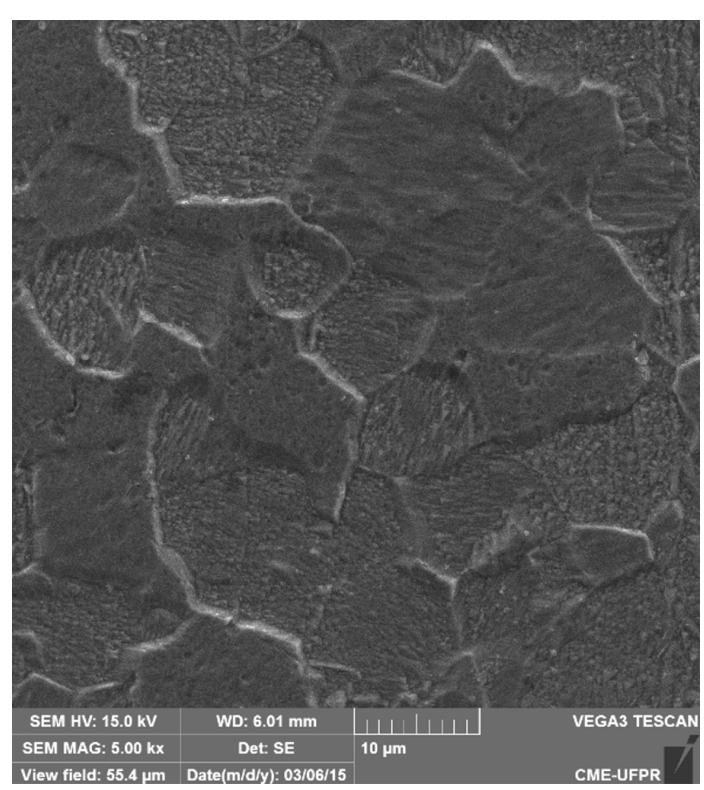

(a)

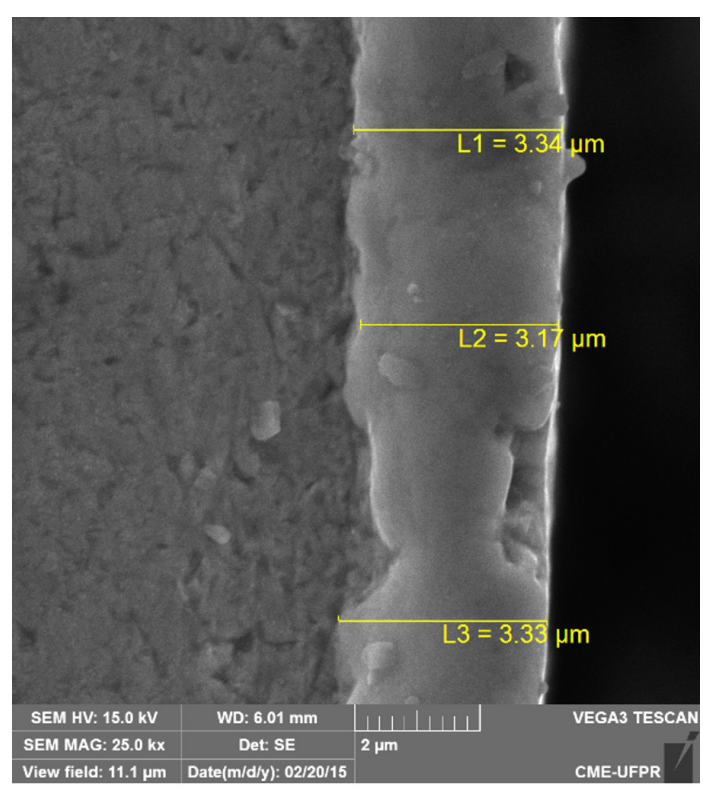

(b)

Figure 4. Cross-section SEM micrography of the: (a) niobium substrate bulk, before nitriding; and (b) nitrided layer, obtained after $4 \mathrm{~h}$ $(2+2 \mathrm{~h})$ nitriding treatment.

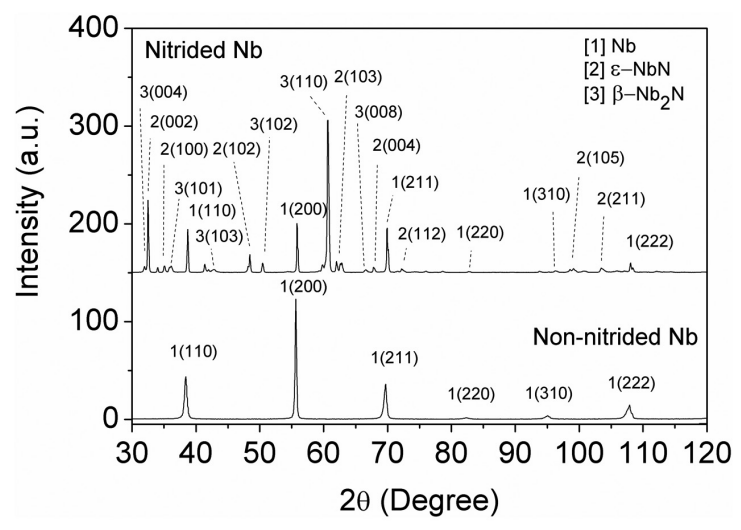

Figure 5. The XRD pattern of the nitrided and non-nitrided niobium surface.

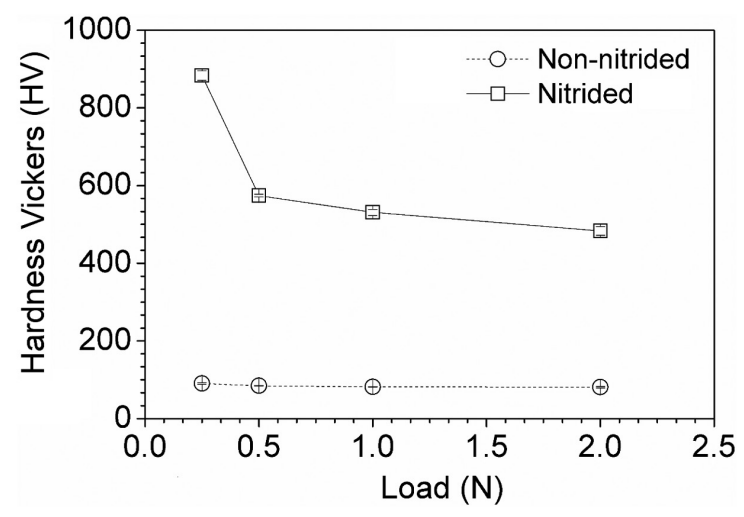

Figure 6. Hardness values of the studied surfaces obtained by using the Vickers indentation technique as a function of the load. niobium substrates. On the other hand, strong variation in the measured values of the nitrided surface can be observed by decreasing the used load from 2 to $0.25 \mathrm{~N}$ load. This result is due to the influence of the chemically non-altered niobium substrate bulk (which is very ductile) on the nitrided surface hardness measurement, since the obtained nitrided layer is too thin, as shown in Figure $4 \mathrm{~b}$. Thus the value on the order of $900 \mathrm{HV}$ obtained using $0.25 \mathrm{~N}$ load is far from being the true hardness of the nitrided surface. In Figure 7, the brittle feature of the studied system formed by 'ceramic $(\mathrm{NbN})$ layer + metallic $(\mathrm{Nb})$ bulk' can be evidenced by the presence of cracks around the Vickers indentation performed at $2 \mathrm{~N}$ loading. In this case, SEM micrography of the indicated indentation (in the center, at the left) was detailed by using higher magnitude, and secondary electrons (at the top), and backscattered electrons (at the bottom) detectors. Aiming to determine the nitrided surface hardness closer to the true value, nanoindentation technique was also performed. Figure $3 \mathrm{a}, \mathrm{b}$, show the nanohardness, and elastic modulus results as a function of the indentation (penetration) depth, for the studied surfaces, respectively. The nanoindentation characterization of the surface in the as-nitrided condition was not possible to be achieved, since values close to zero were obtained, as shown in Figure 3a. These unreal results were due to the high roughness of the nitrided surface (see Figure 1b, and Table 1 results). It is to be noted that the problem of nanoindentation characterization in rough surfaces attained by plasma nitriding is well known, and it has been extensively stressed in ${ }^{15}$. Aiming to turn this problem, the original nitrided surface was repolished, and a new topography was obtained showing lower roughness values (see Table 1). This procedure made possible to estimate maximum nitrided surface hardness values on the order of $18 \mathrm{GPa}$, on average, at $500 \mathrm{~nm}$ penetration depth, 
which agrees well with results previously presented in $^{3}$, considering the differences of nitriding conditions in both the works. For comparison purpose, it is to be noted that $1 \mathrm{GPa}$ is usually correlated to be equal to $100 \mathrm{HV}$.

In addition, the empirical rule for which the substrate bulk effect on the measured hardness of a hard film tends to be negligible for small indenter penetration depths, usually smaller than $10-20 \%$ of the total film thickness, in accordance with $^{16}$, was confirmed here. In this case, the measured nitrided layer thickness value of $3.3 \mu \mathrm{m}$ (as shown in Figure $4 \mathrm{~b}$ ) is attained to the condition for which the penetration depth of $500 \mathrm{~nm}$ would correspond to $15 \%$ of the total nitrided layer thickness. On the other hand, the lower hardness values ranging from 11 to $18 \mathrm{GPa}$, at shallow tip penetrations $(<500 \mathrm{~nm})$, are also due to the nitrided surface roughness. Finally, for penetration depths $>500 \mathrm{~nm}$, a continuous decrease to hardness values near $5 \mathrm{GPa}$ is verified for contact depths up to $2580 \mathrm{~nm}$. In Figure $3 \mathrm{~b}$, the elastic modulus determination shows a decrease on average on the order of 360 (ranging from $450 \mathrm{GPa}$ maximum value to $250 \mathrm{GPa}$ minimum value) to $120 \mathrm{GPa}$ range, by varying the penetration depth from 170 to $2580 \mathrm{~nm}$, respectively. For comparison purpose, it is to be noted that nanocrystalline $\mathrm{NbN}$ films has shown high hardness (36.6 GPa) and a high elastic modulus (457 GPa) and a good elastic recovery $(71 \%)$, as presented in ${ }^{17}$. On the other hand, non-nitrided niobium has presented hardness, and elastic modulus on the order of $1 \mathrm{GPa}$, and $100 \mathrm{GPa}$, respectively (Figure 5), which agree well with the expected for pure niobium ${ }^{18,19}$. The whole of properties obtained for the nitrided samples in this work, as above mentioned, confirms that very hard and thin surfaces, thus apparently presenting low ductility, were obtained in present case.

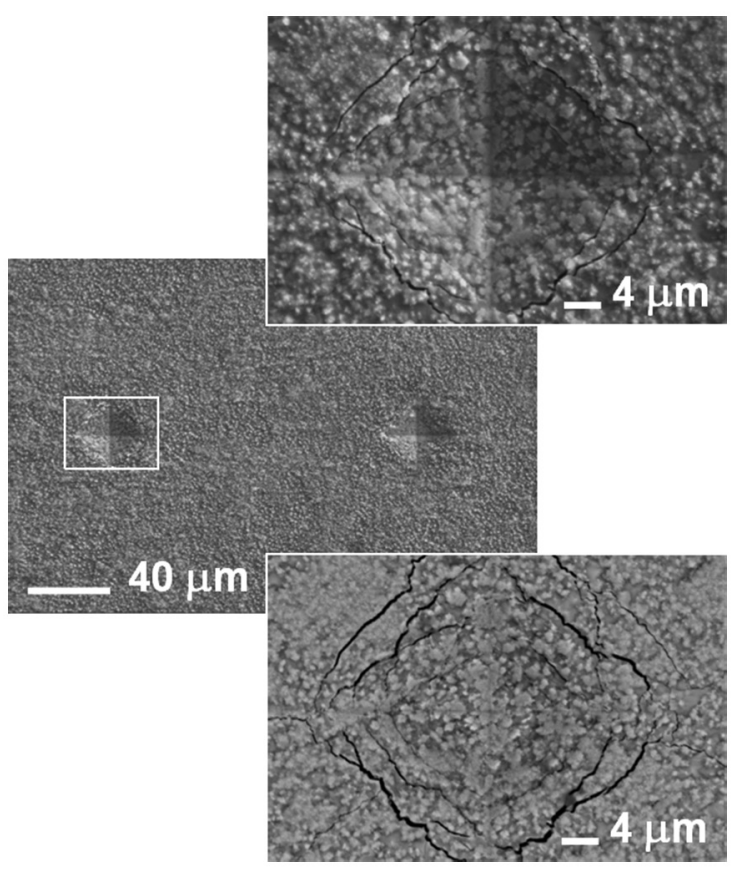

Figure 7. SEM image of microhardness (Vickers) indentations performed at $2 \mathrm{~N}$ load in the nitrided niobium surface (in central). Indentation detail attained by using secondary (at the top), and backscattered electron (at the bottom) detectors.

\subsection{Cavitation erosion behavior characterization of the system formed by 'ceramic ( $N b N)$ \\ layer + metallic $(\mathrm{Nb})$ bulk'}

Figure 8 presents the cumulative mass loss (CML) curve as a function of the cavitation erosion time for non-nitrided and nitrided niobium. It is evidenced that nitrided niobium surfaces, comprising the system formed by 'ceramic $(\mathrm{NbN})$ layer + metallic $(\mathrm{Nb})$ bulk' in study, tend to present higher cavitation resistance than at the non-nitrided niobium. For the case of annealed niobium, as presented $\mathrm{in}^{2}$, the energy provided by the impacts of microjets and/or spherical collapse of micro bubbles would be spent in higher proportion to produce plastic deformation work on the specimen surface, resulting in the work-hardening effect, which necessarily precedes the crack formation process in materials typically ductile like niobium. On the other hand, for the nitrided niobium surface, the formation of niobium nitride phases $\left(\mathrm{NbN}-\varepsilon\right.$ and $\left.\mathrm{Nb}_{2} \mathrm{~N}-\beta\right)$ has necessarily led to a strong strengthening of the surface, when confronted with its original state, before nitriding, as verified by the Figure $3 \mathrm{a}$, b nanoindentation characterization results. In this case, the whole of properties obtained for the nitrided samples in this work confirms that very hard and thin nitrided layer surfaces, thus apparently presenting low ductility, were obtained. Since the hexagonal niobium nitride phase presents high covalent character, such material tend to show no plastic deformation when submitted to mechanical loading, thus the main way to relieve the imposed stresses due to the bubbles collapse, in cavitation erosion testing, would be by nucleating cracks next to surface. As seen, the results of Vickers indentation test (see Figure 7) using $2 \mathrm{~N}$ (200 gf) load on the niobium nitrided surface confirms the assumption that the nitrided layer in niobium substrates tends to present low ductility, at least for the conditions studied here. In the case of Figure 7, the damaged surface characterized in details by SEM images using as secondary electrons as backscattered electrons detectors, clearly shows the occurrence of microcracks, which apparently show no plastic deformation. It is to be noted that cracking

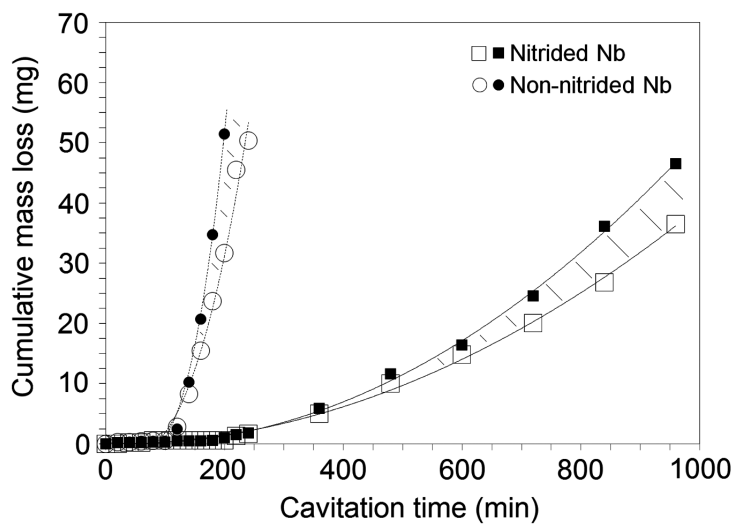

Figure 8. The cumulative mass loss (CML) curve as a function of the cavitation erosion time for non-nitrided and nitrided niobium surfaces (Fulfilled-solid icons are referred to the best results; non-fulfilled icons are referred to the worst one; and dashed lines indicate the variation field of the measurements). 
and spalling are the predominant modes of wear for hard brittle materials, according to Stachowiak ${ }^{1}$, being the case of the surface system studied here. The SEM images of the cavitation eroded surface of a nitrided niobium sample tested for a time of $180 \mathrm{~min}$, and $960 \mathrm{~min}$ are presented in Figure 9a, b, respectively. Figure 9a shows a typical surface morphology in the incubation stage, presenting few and too small pits formation (black-aspect points), and no apparent undulations. In this case, the single impact loads tend to remove some and small sharply edged nanoparticles during the incubation stage, being that the sites in which such particles are extracted seem to coincide with the original positions of the island-like products nucleated at the surface. Eventually craters formed in the first incubation period cycles can also be present (results not-shown) confirming that advanced material removal can be verified in very small and specific sites in the initial times of the cavitation testing. On the other hand, craters formed in profusion all over the nitrided surface, eroded at the 960 min cavitation testing time, can be seen in Figure $9 \mathrm{~b}$. In this case, the appearance of fatigue striations inside the craters, in specific sites, similar to verified $i^{2}$, is strong indicative that the nitrided (ceramic phase) layer was completely removed from the surface by cavitation erosion at the longest testing time (see Table 2). This assumption is based on the fact that the nitride layer tends to fail in brittle mode, as expected for ceramic phases, thus at the absence of fatigue fail mode, which is the typical cavitation erosion mechanism of metals.

Table 2 summarizes the results obtained to determine the cumulative mass loss (CML), and the average erosion rate
(AER) as a function of the cavitation erosion testing time for non-nitrided and nitrided niobium samples. The CML and AER values were $43.9 \mathrm{mg}$ and $14.6 \mathrm{mg} / \mathrm{h}$ for non-nitrided niobium, and $0.6 \mathrm{mg}$ and $0.2 \mathrm{mg} / \mathrm{h}$ for nitrided niobium, respectively.

These results indicate that non-nitrided niobium has reached the maximum erosion rate stage, as verified $i^{2}$, while the nitrided niobium is in the incubation stage. On the other hand, CML and AER values of $44.5 \mathrm{mg}$ and $2.78 \mathrm{mg} / \mathrm{h}$, respectively, were determined for the nitrided niobium at 960 min cavitation testing time. In this case, the strong inclination difference of both the cavitation erosion curves of the Figure 8 results, for testing times longer than the incubation time of each case, confirm that the system 'ceramic layer + metallic bulk' cavitation erosion behavior tends to be slightly changed from the accelerate stage to the maximum erosion rate stage, after the incubation period. Such behavior shall be associated to the fact that the niobium nitride presents elastic modulus of about 4 times higher than the metallic niobium. In this case, the nitrided layer tends to respond elastically to the impacts of microjets and/or spherical collapse of bubbles and this would explain its better behavior to cavitation erosion testing. Finally, from the Figure 8 results, it can be determined that the nominal incubation period (IP) of the nitrided niobium (on the order of $200 \mathrm{~min}$ ) was at least twice higher than that verified for non-nitrided niobium (of about $90 \mathrm{~min}$ ), for the nitriding conditions studied here.

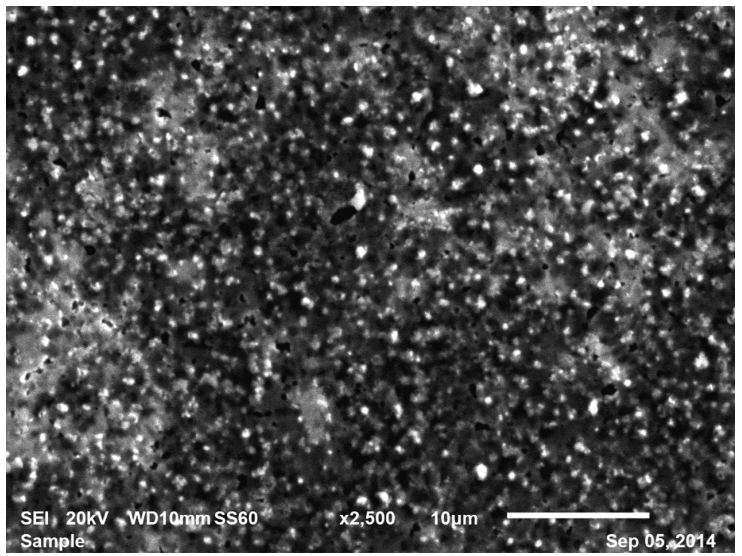

(a)

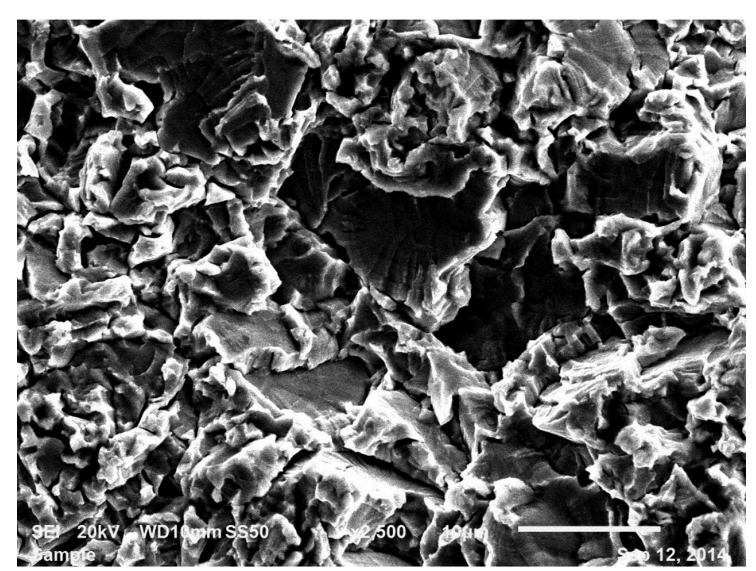

(b)

Figure 9. SEM images of the cavitation eroded nitrided surface at: (a) $180 \mathrm{~min}$; and (b) $960 \mathrm{~min}$ testing time.

Table 2. Cumulative mass loss (CML) and the average erosion rate (AER) values as a function of the cavitation erosion testing time for non-nitrided and nitrided niobium samples.

\begin{tabular}{|c|c|c|c|c|c|c|c|c|c|c|c|c|c|c|c|c|c|c|c|c|}
\hline \multirow{2}{*}{ Parameter } & \multirow{2}{*}{ Nb condition } & \multicolumn{19}{|c|}{ Cavitation Time (min) } \\
\hline & & 0 & 20 & 40 & 60 & 80 & 100 & 120 & 140 & 160 & 180 & 200 & 220 & 240 & 360 & 480 & 600 & 720 & 840 & 960 \\
\hline \multirow{2}{*}{ CML (mg) } & Non-nitrided & 0 & 0 & 0 & 0 & 2.3 & 8.1 & 16.4 & 23.1 & 32.6 & 43.9 & - & - & - & - & - & - & - & - & - \\
\hline & Nitrided & 0 & 0.2 & 0.2 & 0.2 & 0.3 & 0.3 & 0.5 & 0.5 & 0.5 & 0.6 & 1.0 & 1.5 & 1.8 & 6.9 & 13.3 & 17.4 & 24.6 & 34.1 & 44.5 \\
\hline \multirow{2}{*}{$\operatorname{AER}(\mathrm{mg} / \mathrm{h})$} & Non-nitrided & 0 & 0 & 0 & 0 & 1.7 & 3.0 & 8.2 & 9.9 & 12.2 & 14.6 & - & - & - & - & - & - & - & - & - \\
\hline & Nitrided & 0 & 0.6 & 0.3 & 0.2 & 0.2 & 0.1 & 0.25 & 0.2 & 0.19 & 0.2 & 0.3 & 0.41 & 0.45 & 1.55 & 1.66 & 1.74 & 2.05 & 2.43 & 2.78 \\
\hline
\end{tabular}




\section{Conclusion and Final Remarks}

The first results of the plasma nitriding study performed in pure niobium in order to improve its cavitation erosion behavior were presented in this work. Emphasis was given to determine the cavitation erosion behavior of the system 'ceramic layer + metallic bulk', by confronting it with the metallic system behavior, in the case the non-nitrided (or pure) niobium. The main conclusions can be listed as follows:

- Plasma nitriding process successfully leads the niobium substrate to have improved its cavitiation erosion behavior;

- The increase of the cavitiation erosion resistance is attributed to the whole of mechanical and metallurgical properties of the obtained system 'ceramic layer + metallic bulk', which is constituted of $\mathrm{NbN}-\varepsilon$ and $\mathrm{Nb}_{2} \mathrm{~N}-\beta$ phases present in the ceramic layer, and niobium in the metallic bulk;

- The cavitation erosion behavior of the system 'ceramic layer + metallic bulk' tends to present a slight change from the accelerate stage to the maximum erosion rate stage, after the incubation period, when compared to the non-nitrided niobium;

\section{References}

1. Stachowiak GW and Batchelor AW. Abrasive, erosive and cavitation wear engineering tribology. 3rd ed. Boston: Butterworth-Heinemann; 2006.

2. Brunatto SF, Allenstein AN, Allenstein CLM and Buschinelli AJA. Cavitation erosion behavior of niobium. Wear. 2012; 274-275:220-228. http://dx.doi.org/10.1016/j.wear.2011.09.001

3. Borcz C, Lepienski CM and Brunatto SF. Surface modification of pure niobium by plasma nitriding. Surface and Coatings Technology. 2013; 224:114-119. http://dx.doi.org/10.1016/j. surfcoat.2013.03.008.

4. Santos JF, Garzón CM and Tschiptschin AP. Improvement of the cavitation erosion resistance of an austenitic AISI 304L stainless steel by high temperature gas nitriding. Materials Science and Engineering: A. 2004; 382:378-386.

5. Allenstein AN, Lepienski CM, Buschinelli AJA and Brunatto SF. Improvement of the cavitation erosion resistance for lowtemperature plasma nitrided CA-6NM martensitic stainless steel. Wear. 2014; 309(1-2):159-165. http://dx.doi.org/10.1016/j. wear.2013.11.002.

6. Tomlinson WJ and Matthews SJ. Cavitation Erosion of Structural Ceramics. Ceramics International. 1994; 20(3):201-209. http:// dx.doi.org/10.1016/0272-8842(94)90040-X.

7. Niebuhr D. Cavitation erosion behavior of ceramics in aqueous solutions. Wear. 2007; 263(1-6):295-300. http://dx.doi. org/10.1016/j.wear.2006.12.040.

8. Fatjó GGA, Hadfield M and Tabeshfar K. Pseudoplastic deformation pits on polished ceramics due to cavitation erosion. Ceramics International. 2011; 37(6):1919-1927. http://dx.doi. org/10.1016/j.ceramint.2011.03.043.

9. Pedzich Z, Jasionowski R and Ziabka M. Cavitation wear of structural oxide ceramics and selected composite materials. Journal of the European Ceramic Society. 2014; 34(14):33513356. http://dx.doi.org/10.1016/j.jeurceramsoc.2014.04.022.
- The character more resilient of the nitrided layer in relation to the non-nitrided niobium, evidenced here, opens up a new and promising field for niobium research; and, finally,

- Additional efforts are being conducted in the present moment, aiming to elucidate the cavitation erosion fail mechanism of the system 'ceramic layer + metallic bulk' obtained in this work.

\section{Acknowledgements}

This work was supported by CNPq, Fundação Araucária of the Paraná State, CNPq-Universal Grant N. 482380/2012-8, and MCTI/CNPq/CT-Aquaviário Grant N. 456347/2013-5. The authors also wish to express their thanks to the Laboratory of X-ray Optics and Instrumentation (LORXI) and Laboratory of Minerals (LAMIR) from UFPR, by the use of the XRD and SEM equipments, and to Dr. Sara Blunk by performing the nanoindentation characterization. Thanks are also due to Dr. Julio Klein Neves, and Dr. Giuseppe Pintaúde from UTFPR, by the use of SEM and the high precision 3D profile analysis equipment.

10. Karunamurthy B, Hadfield M, Vieillard C and Morales G. Cavitation erosion in silicon nitride: Experimental investigations on the mechanism of material degradation. Tribology International. 2010; 43(12):2251-2257. http://dx.doi. org/10.1016/j.triboint.2010.06.012.

11. Karunamurthy B, Hadfield M, Vieillard C, Morales-Espejel $\mathrm{GE}$ and Khan Z. Cavitation and rolling wear in silicon nitride. Ceramics International. 2010; 36(4):1373-1381. http://dx.doi. org/10.1016/j.ceramint.2010.02.011.

12. Krella AK. The new parameter to assess cavitation erosion resistance of hard PVD coatings. Engineering Failure Analysis. 2011; 18(3):855-867. http://dx.doi.org/10.1016/j. engfailanal.2010.10.002.

13. Krella A and Czyzniewski A. Influence of the substrate hardness on the cavitation erosion resistance of TiN coating. Wear. 2007; 263(1-6):395-401. http://dx.doi.org/10.1016/j.wear.2007.02.003.

14. Chapman B. Glow discharge processes. New York: John Wiley \& Sons; 1980 .

15. Souza GB, Foerster CE, Silva SLR and Lepienski CM. Nanomechanical properties of rough surfaces. Materials Research. 2006; 9(2):159-163. http://dx.doi.org/10.1590/ S1516-14392006000200009.

16. Foerster CE, Assmann A, Silva SLR, Nascimento FC, Lepienski CM, Guimarães JL, et al. AISI 304 nitrocarburized at low temperature: Mechanical and tribological properties. Surface and Coatings Technology. 2010; 204(18-19):3004-3008. http:// dx.doi.org/10.1016/j.surfcoat.2009.12.030.

17. Han Z, Hu X, Tian J, Li G and Mingyuan G. Magnetron sputtered $\mathrm{NbN}$ thin films and mechanical properties. Surface and Coatings Technology. 2004; 179(2-3):188-192. http:// dx.doi.org/10.1016/S0257-8972(03)00848-X.

18. American Society for Microbiology - ASM. Properties and selection: nonferrous alloys and special-purpose materials. 10th ed. Ohio; 1990. ASM Handbooks, v. 2.

19. Wilkinson WD. Fabrication of refractory metals. New York: Gordon and Breach Science Publishers Inc.; 1970. 